\title{
The Body Inversion Effect Is Mediated by Face-Selective, Not Body-Selective, Mechanisms
}

\author{
Talia Brandman and Galit Yovel \\ Department of Psychology, Tel Aviv University, Tel Aviv, 69978, Israel
}

Evidence suggesting that the brain has specialized mechanisms for processing human bodies include the discovery of body-selective brain areas and the behavioral body inversion effect (BIE). Interestingly, the BIE (worse discrimination of inverted vs upright bodies) disappears for headless bodies, implying a critical role of the head in this effect. Previous studies have shown that the face inversion effect is mediated by the fusiform face-selective area. Given the central role that the head plays in the behavioral BIE, we asked whether it is mediated by face-selective or body-selective areas. In two event-related functional magnetic resonance-adaptation experiments, we examined the representation of upright and inverted bodies in category-selective occipitotemporal areas. In the first experiment we presented whole (faceless) bodies, while in the second we presented headless bodies. Both experiments consisted of pairs of upright and inverted bodies that were either the same or different in posture. Body-selective areas showed similar adaptation effects for upright and inverted whole or headless bodies, suggesting similar discrimination for the two orientations regardless of the head. In contrast, faceselective areas showed an adaptation effect to upright but not inverted bodies, and for whole but not for headless bodies. Thus, the response of the face-selective, but not body-selective areas, is consistent with the behavioral BIE in that it shows better discrimination for upright than inverted bodies, for whole but not for headless bodies. These results suggest a critical role for the head in the processing of human bodies.

\section{Introduction}

Recent studies have demonstrated that human bodies are processed by specialized neural and cognitive mechanisms. Specifically, functional magnetic resonance ( $\mathrm{fMR}$ ) imaging (fMRI) studies have uncovered two body-selective brain areas: the extrastriate body area (EBA) in the occipital cortex and the fusiform body area (FBA) in the fusiform gyrus (for review, see Peelen and Downing, 2007). These areas are characterized by a highly selective response to whole bodies, headless bodies and body parts over any other object-category stimuli (faces, houses, other objects, etc.) (Downing et al., 2001). Further evidence for specialized mechanisms for processing human bodies is the body inversion effect (BIE), in which recognition performance of inverted bodies is inferior to that of upright bodies. The BIE has been shown to be as large as the well established face inversion effect (Reed et al., 2003; Minnebusch et al., 2009; Yovel et al., 2010), and considerably larger than the inversion effect for any other object category (Reed et al., 2003, 2006). The extensively studied face inversion effect has long been considered a marker for specialized mechanisms for the processing of upright faces (Young et al., 1987; Farah et al., 1998; Maurer et al., 2002; Tanaka and Farah, 2003), and has been shown to be associated with face-selective brain areas (Yovel and Kanwisher,

\footnotetext{
Received Feb. 9, 2010; revised May 5, 2010; accepted June 4, 2010.

This study is supported by a grant from the Israeli Science Foundation 65/08 to G.Y. and an Adams Super Center for Brain Studies Fellowship to T.B. We thank Tatiana Pelc for help with stimulus generation. We also thank Sharon Gilad, Vadim Axelrod, and Boaz Sadeh for valuable comments on this manuscript.

Correspondence should be addressed to Galit Yovel, Department of Psychology, Tel Aviv University, Tel Aviv, 69978, Israel. E-mail: galit@freud.tau.ac.il.

DOI:10.1523/JNEUROSCI.0911-10.2010

Copyright $\odot 2010$ the authors $\quad$ 0270-6474/10/3010534-07\$15.00/0
}

2005; Mazard et al., 2006). However, little is known about the neural basis of the BIE.

Given the association of the face inversion effect with faceselective brain areas, a reasonable prediction would be that the BIE is mediated by body-selective brain areas. However, the only study to have explicitly examined a possible association between the BIE and body-selective brain areas have shown that transcranial magnetic stimulation (TMS) of the EBA increased the BIE by decreasing performance for inverted but not upright bodies, suggesting that the BIE is not mediated by the EBA (Urgesi et al., 2007). Another possible hypothesis is that the BIE may be mediated by the FBA, given that both have been associated with holistic processing of human bodies (Reed et al., 2006; Taylor et al., 2007). This hypothesis cannot be examined with TMS due to the deep location of the FBA and should be therefore addressed with fMRI. Finally, given recent reports that the behavioral BIE is abolished for headless bodies (Minnebusch et al., 2009; Yovel et al., 2010), it is possible that face/head-selective areas may be associated with the BIE, a hypothesis that has not been tested yet.

To find out whether the BIE is mediated by face and/or bodyselective mechanisms, we conducted a fMR-adaptation study (Grill-Spector and Malach, 2001) in which we measured the fMRI response of body and face-selective areas to pairs of images of bodies that were either the same or differed by body posture. A higher response to different versus same stimuli (i.e., adaptation effect) indicates that a particular area is involved in discriminating between the two different stimuli (Grill-Spector et al., 2006). Accordingly, a neural correlate for a BIE would be a stronger adaptation effect to upright than inverted bodies. 


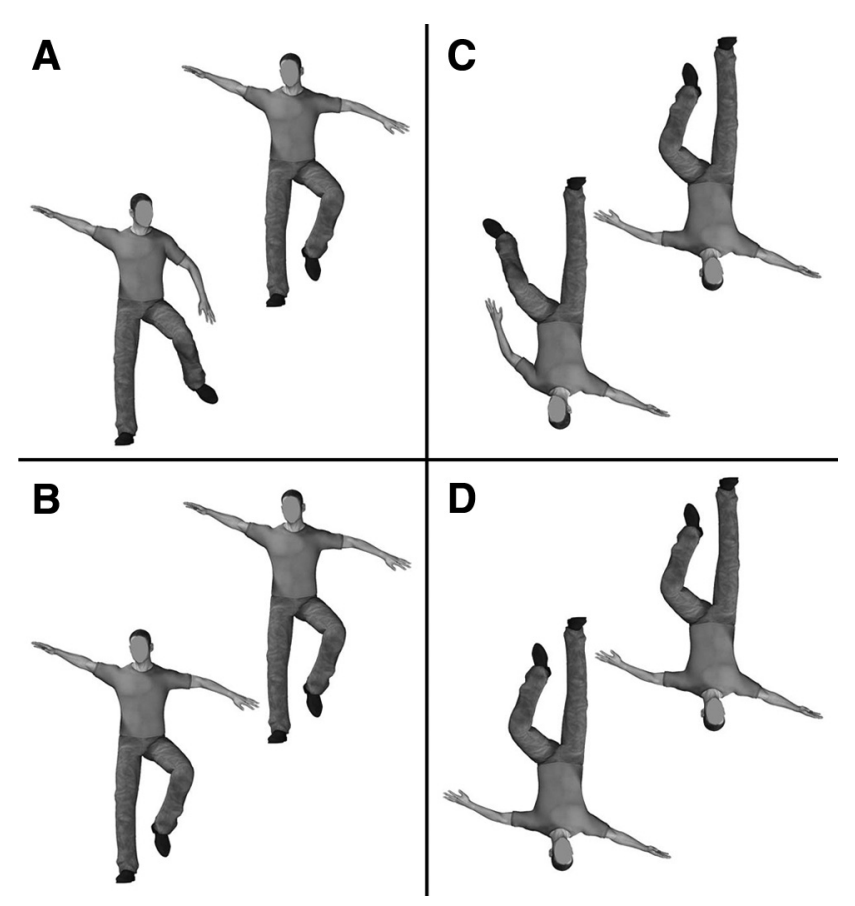

Figure 1. The fMR-adaptation task in experiment I included four types of pairs of whole bodies. $\boldsymbol{A}$, Upright, different posture; $\boldsymbol{B}$, upright, same posture; $\boldsymbol{C}$, inverted, different posture; $\boldsymbol{D}$, inverted, same posture.

\section{Materials and Methods}

\section{Subjects}

Eleven healthy volunteers (6 males, ages 19-28) with normal or corrected to normal vision participated in experiment I. One subject was excluded from data analysis due to excessive head motion during scanning. Fourteen healthy volunteers ( 9 females, ages 22-35) that had not participated in experiment I, participated in experiment II. Two subjects were excluded due to data loss (scanner failure), and two more were excluded from the data analysis due to excessive head motion during scanning, leaving a total of 10 subjects in the final analysis ( 6 females). All subjects gave informed consent to participate in the study, which was approved by the ethics committee of the Tel Aviv Sourasky Medical Center.

\section{Stimuli}

Stimuli of the functional localizer were 80 grayscale photographs of headless bodies, faces, objects, and scrambled objects (i.e., a random mixture of pixels of each of the object images). The stimuli of the fMR-adaptation sessions in experiment I consisted of 16 computer-generated grayscale images of human body figures created with Poser 7 (Smith Micro Software) and processed in Adobe Photoshop CS2. The figures differed by posture of the head, arm and leg and were presented in an upright or inverted orientation, thus creating a total of 16 distinct pairs. All poses were physically possible, nonmeaningful actions (Fig. 1). Stimuli in experiment II included headless versions of the 16 body images used in experiment I (Fig. 2). Heads were removed from the original images using Adobe Photoshop CS2. We presented exactly the same stimuli for each different and same pairs as in experiment I.

\section{Apparatus and procedure}

High-resolution fMRI data were collected in a 3T GE MRI scanner, using an 8-channel coil. Echoplanar imaging sequence was used to collect fMRI data with a TR of $2 \mathrm{~s}$, TE of $35 \mathrm{~ms}, 23$ or 26 slices per TR, slice thickness of $2.4 \mathrm{~mm}$ no-gap (matrix $96 \times 96$ ) and FOV of $20 \mathrm{~cm}$. Stimuli were presented with Matlab (Psychtoolbox) (Brainard, 1997), and projected onto a screen viewed by the subjects through a mirror located in the scanner.

Functional localizer included four object categories presented in a blocked design: bodies, faces, objects and scrambled objects. Each block lasted $16 \mathrm{~s}$ and included 20 stimuli, each presented for $350 \mathrm{~ms}$ with a $450 \mathrm{~ms}$ interstimu-

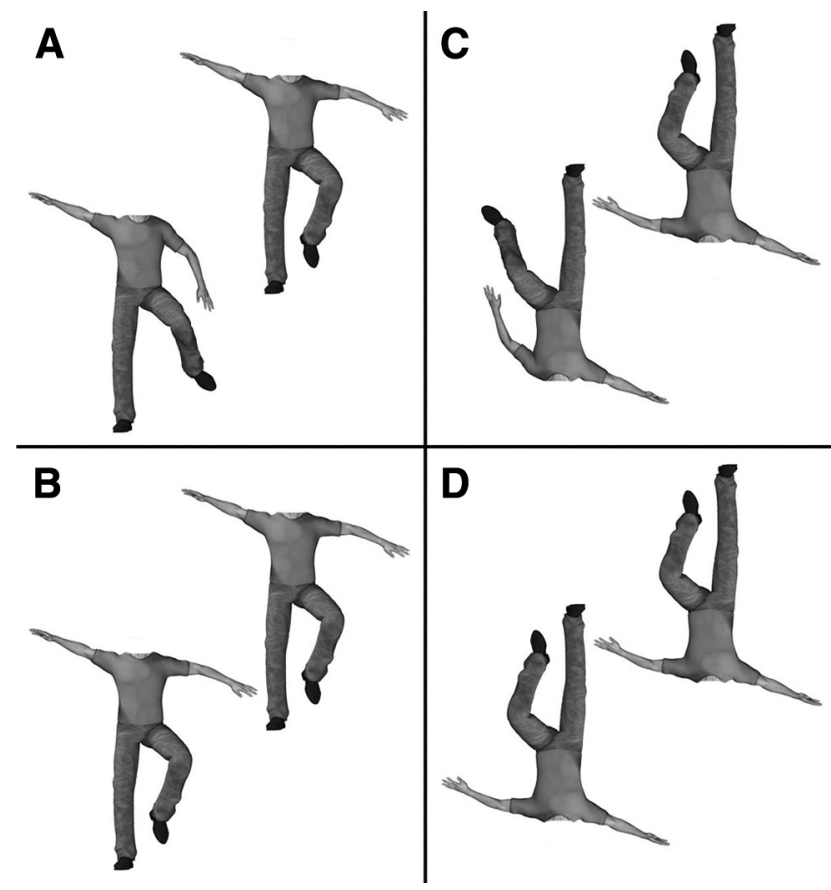

Figure 2. The fMR-adaptation task in experiment II included four types of pairs of headless bodies. $\boldsymbol{A}$, Upright, different posture; $\boldsymbol{B}$, upright, same posture; $\boldsymbol{C}$, inverted, different posture; $\boldsymbol{D}$, Inverted, same posture.

lus interval. Category block order was counterbalanced within and across sessions. Each localizer session consisted of 4 blocks for each category and 5 blocks of a baseline fixation point (a total of $336 \mathrm{~s}$ ). To maintain vigilance, subjects were instructed to press a response box button, whenever two identical images appeared consecutively (a 1-back task).

In the main experiment we presented pairs of bodies in a rapid eventrelated paradigm. On each trial two body images were presented sequentially, each for $1 \mathrm{~s}$, with a $200 \mathrm{~ms}$ interstimulus interval and a $1.8 \mathrm{~s}$ intertrial interval. The stimuli were presented in four conditions of a randomly mixed design: pairs of same or different body posture, in an upright or inverted orientation. Each session included 16 trials in each condition ( $4 \mathrm{~s}$ per trial) and a total of $64 \mathrm{~s}$ of baseline fixation point $(320$ $s$ total). The order of the four experimental conditions and the blank trials was determined by an algorithm that generates an optimal sequence for extraction of hemodynamic responses for rapid event related trials (http://surfer.nmr.mgh.harvard.edu/optseq/). For each pair of consecutive images, the subject was asked to press one key if they were identical, or another if they were different in posture. Responses were collected to assess the behavioral effects of this sequential matching task in addition to the measured fMR-adaptation.

\section{Data analysis}

fMRI data analysis was accomplished using statistical parametric mapping (SPM5). The first six volumes were acquired during a blank screen display and were discarded from the analysis. The data were then preprocessed using slice timing correction, realignment and spatial smoothing with a $3 \times 3 \times 3 \mathrm{~mm}$ full-width at half-maximum (FWHM) Gaussian kernel. A GLM HRF model was estimated for each subject.

ROI-analysis. In the localizer experiment, category-selective voxels were defined using combinations of $t$-contrasts to assure their specific selectivity among all 4 categories as well as their positive BOLD response to the selected category. Body-selective areas EBA and FBA were extracted from a full conjunction of bodies $>$ objects, body $>$ scrambled-objects and body $>0 t$ contrast maps $(p<0.00001)$, masking out voxels of faces $>$ objects $t$-contrast map $(p<0.05)$. Similarly, face-selective areas FFA and OFA were extracted from a full conjunction of faces $>$ objects, faces $>$ scrambledobjects and faces $>0 t$-contrast maps $(p<0.00001)$, excluding bodies $>$ objects $t$-contrast $(p<0.05)$. Finally, object-selective areas of the LOC (LO 
and $\mathrm{pFs}$ ) were extracted from a full conjunction of objects $>$ scrambledobjects and objects $>0 t$-contrast maps $(p<0.00001)$, with exclusion masking of both bodies $>$ objects and faces $>$ objects $t$-contrasts $(p<0.05)$. ROIs include only voxels that passed the significance level for each contrast.

Main experiment. Time courses were extracted for each of the four conditions using the MarsBaR region of interest toolbox for SPM within each of the predefined ROIs. The-dependent measure was the peak value of time courses (3-4 TRs from trial onset). Statistical analysis of the peak response to each of the four body conditions within each ROI, as well as the behavioral task responses were performed with Statistica 7.

\section{Results}

\section{Experiment I-Inversion effect for whole bodies}

Behavioral results

Behavioral responses collected in the scanner replicated previous findings (Reed et al., 2003; Minnebusch et al., 2009; Yovel et al., 2010). Accuracy rates were higher for upright (97\%) than inverted bodies (94\%). A paired $t$ test revealed a significant difference between upright and inverted conditions $\left(t_{(9)}=3.14, p=0.012\right)$. In addition, response time was higher for inverted (703.0 ms) than upright bodies (639.5 ms). A paired $t$ test revealed a significant difference between upright and inverted conditions $\left(t_{(9)}=3.18, p=0.011\right)$.

\section{$f M R$-adaptation results}

Body-selective areas. A repeated-measures ANOVA with area (EBA, FBA), hemisphere (left, right), orientation (upright, inverted) and adaptation (same, different) as within-subject factors, revealed no effects or interactions with hemisphere $(p>0.18)$. Therefore, data were collapsed across hemispheres using a weighted averaged based on the relative volumes of the body-selective areas in each hemisphere.

Both the EBA and FBA showed similar discrimination for upright and inverted bodies. The magnitude of the adaptation effect (i.e., higher response to different relative to same pairs) did not differ between upright and inverted bodies (Fig. 3A; supplemental Fig. $1 A$, available at www.jneurosci.org as supplemental material). Furthermore, body-selective areas showed a higher response to inverted than upright bodies. A repeated-measures ANOVA of the body-selective areas, with area, orientation and adaptation as within-subject factors, revealed a main effect of adaptation $\left(F_{(1,9)}=14.67, p=0.004\right)$, a main effect of orientation $\left(F_{(1,9)}=9.65, p=0.012\right)$ and no interaction between them $(p>0.4)$. No other interactions were significant.

Face-selective areas. Interestingly, unlike the body-selective areas, face-selective areas (FFA and OFA) do exhibit better discrimination for upright than inverted bodies. The magnitude of adaptation effect was larger for upright than inverted bodies (Fig. $3 B$; supplemental Fig. $1 B$, available at www.jneurosci.org as supplemental material). A repeated-measures ANOVA of the faceselective areas, with area (FFA, OFA), hemisphere, orientation and adaptation as within-subject factors, revealed an interaction between adaptation and orientation $\left(F_{(1,8)}=7.68, p=0.024\right)$. Paired $t$ test showed higher response to different from same pairs for upright bodies $\left(t_{(8)}=3.74, p=0.006\right)$ but not inverted bodies $(p>0.5)$. A significant interaction was also found between area and hemisphere $\left(F_{(1,8)}=10.12, p=0.013\right)$ with higher response to bodies in the right than the left OFA.

Our results so far show that body and face-selective areas generate different representations to human bodies. We next asked whether the response of any of these regions is unique or similar to the representation that is generated by a general shape processing area, the lateral occipital complex (LOC).

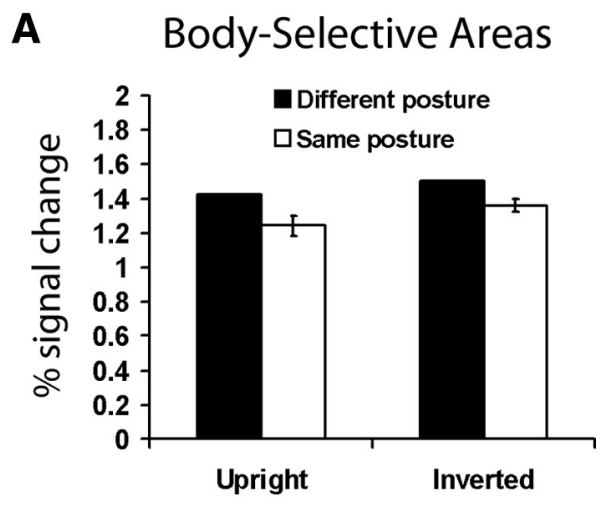

\section{B Face-Selective Areas}
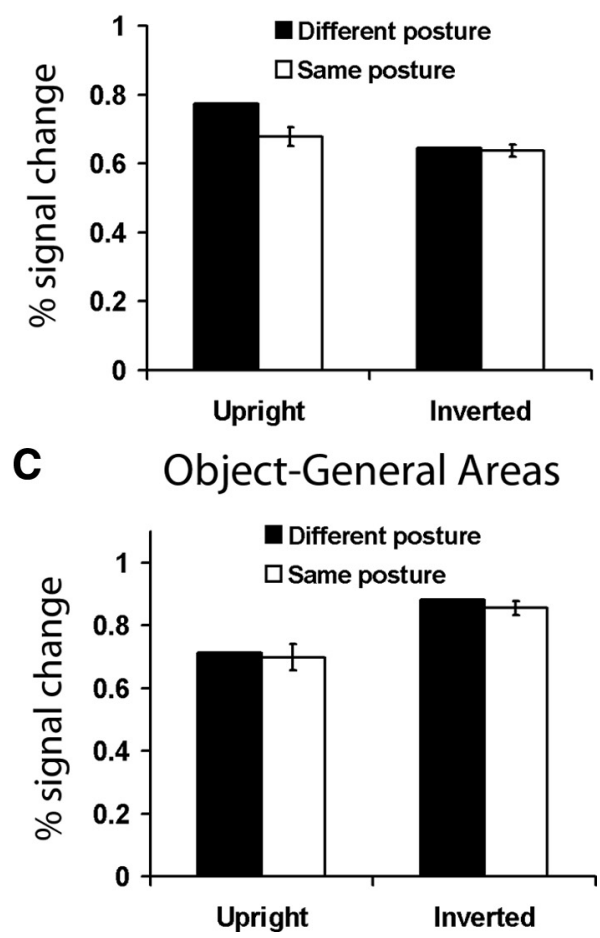

Figure 3. fMR-adaptation to whole bodies. $\boldsymbol{A}$, Body-selective areas showed similar adaptation to inverted and upright bodies, and a higher response to inverted than upright stimuli, but no interaction between adaptation and orientation. Because we found the same pattern of response in the EBA and FBA, the figure presents the data averaged across the two bodyselective areas (see supplemental Fig. 1A, available at www.jneurosci.org as supplemental material for $E B A$ and $F B A$ ). $\boldsymbol{B}$, Face-selective areas showed stronger adaptation to upright than inverted bodies. Because we found the same pattern of response in the OFA and FFA, the figure presents the data averaged across the two face-selective areas (see supplemental Fig. $1 B$, available at www.jneurosci.org as supplemental material for OFA and FFA). C, Object general areas showed higher responses to inverted than upright stimuli and no adaptation to bodies. Because we found the same pattern of response in the $\mathrm{LO}$ and pFs, the figure presents the data averaged across the two object areas (see supplemental Fig. 1C, available at www.jneurosci.org as supplemental material for $\mathrm{LO}$ and $\mathrm{pFs}$ ). Error bars represent the SE of the difference between the responses to different and same stimuli (i.e., the standard error of the adaptation effect).

Object general areas (LOC). The LOC showed a higher response to inverted relative to upright bodies for both same and different pairs, and no discrimination for either upright or inverted bodies (Fig. 3C). A repeated-measures ANOVA of the response of the LOC, with area (LO, pFs), hemisphere, orientation and adaptation as within-subject factors, revealed a main effect of orientation $\left(F_{(1,8)}=\right.$ 57.87, $p=0.00006)$ and no main effect of adaptation $(p>0.3)$. A significant interaction was found between hemisphere and adapta- 
tion $\left(F_{(1,8)}=7.62, p=0.025\right)$ reflects a higher response to different from same bodies in the right-LOC than left-LOC. In addition, interaction between area and orientation $\left(F_{(1,8)}=9.86, p=0.014\right)$ indicates a higher response to inverted than upright bodies in the LO compared with $\mathrm{pFs}$. Finally, main effects of area $\left(F_{(1,8)}=20.65, p=\right.$ $0.002)$ and hemisphere $\left(F_{(1,8)}=90.48, p=0.00001\right)$ indicate higher responses to bodies in the $\mathrm{LO}$ compared with $\mathrm{pFs}$, and in the right compared with left hemisphere.

Overall, our analysis revealed very different pattern of response to upright and inverted bodies in the three object-category-selective areas. To assess whether these patterns significantly differ from one another we proceeded to test interactions between them. For each category-selective area, ventral and lateral, right and left time courses were averaged based on their relative volumes. We performed a repeated-measure ANOVA with category-selective area, orientation and adaptation as within-subject factors. We separately compared the pattern of response in body areas to that in the face areas and in the object areas.

When comparing body to face-selective areas, the three-way interaction between category-selective area (body areas, face areas), adaptation and orientation was not significant $(p=0.12)$. However, separate ANOVA for upright and inverted conditions, with category-selective area (face areas, body areas) and adaptation as within-subject factors, revealed an interaction of categoryselective area and adaptation in the inverted condition $\left(F_{(1,9)}=\right.$ 24.91, $p=0.0006)$ but not in the upright condition $(p>0.3)$. Thus, adaptation to upright bodies was similar in body and faceselective areas, whereas the magnitude of adaptation to inverted bodies was significantly larger in body than in face areas. Furthermore, an interaction was found between area and orientation $\left(F_{(1,9)}=26.16, p=0.0006\right)$, indicating the opposite inversion effects of the body and face-selective areas in their responses to upright versus inverted bodies. The body-selective areas showed a higher response to inverted compared with upright bodies, whereas the face-selective areas showed a higher response to upright compared with inverted bodies.

Comparisons of body versus object-selective areas revealed adaptation in the body areas was significantly larger than in the object areas, as reflected in an interaction between categoryselective area and adaptation $\left(F_{(1,9)}=14.55, p=0.004\right)$. In addition, responses to bodies were higher in the body than the object areas (a main effect of area $F_{(1,9)}=61.48, p=0.00002$ ). Finally, both areas showed a similarly higher response to inverted than upright bodies (a main effect of orientation $F_{(1,9)}=28.83$, $p=0.0004$ and no interaction of orientation and area).

In summary, our findings show that the body-selective areas were as sensitive to upright as to inverted bodies. Thus, their response pattern does not correspond to the behavioral BIE. In contrast, the response of the face-selective areas did correspond to the behavioral BIE, as is evident by the larger adaptation effect to upright bodies than inverted bodies. These findings are similar to adaptation effects that were found for upright and inverted faces in face-selective areas (Yovel and Kanwisher, 2005). Notably, these effects found for body stimuli could not have been directly induced by a face representation, since the faces in all our stimuli were covered. As mentioned above, the behavioral BIE is abolished for headless bodies (Minnebusch et al., 2009; Yovel et al., 2010). It is therefore possible, that the effect that we found in the FFA for whole bodies is mediated by the head. To determine whether the higher sensitivity of the FFA to upright than inverted bodies was induced by the head or whether face-selective areas are directly sensitive to general body posture (without any spe- cific role for head posture), a second fMR-adaptation experiment was conducted using headless body stimuli.

\section{Experiment II-Inversion effect for headless bodies \\ Behavioral results}

Replicating previous findings (Minnebusch et al., 2009; Yovel et al., 2010), no inversion effect was found for headless bodies. Accuracy rates did not differ between upright (97\%) and inverted $(97 \%)$ conditions. A paired $t$ test revealed no difference between upright and inverted conditions $(p>0.7)$. In addition, response time did not differ between inverted $(742.8 \mathrm{~ms})$ than upright bodies $(720.8 \mathrm{~ms})$. A paired $t$ test revealed no difference between upright and inverted conditions $(p>0.26)$.

\section{fMR-adaptation results}

Body-selective areas. A repeated-measures ANOVA with area (EBA, FBA), hemisphere (left, right), orientation (upright, inverted) and adaptation (same, different) as within-subject factors, revealed no effects or interactions of hemisphere $(p>0.3)$. Therefore, data were collapsed across hemispheres using a weighted averaged based on their relative volumes.

Similar to experiment I, body-selective areas EBA and FBA showed an adaptation to headless bodies that did not differ between upright and inverted conditions, and exhibited higher response to inverted than upright bodies (Fig. 4A). A repeated-measures ANOVA on the response of the body-selective areas, with area, orientation and adaptation as within-subject factors, revealed a main effect of adaptation $\left(F_{(1,9)}=8.65, p=0.016\right)$, a main effect of orientation $\left(F_{(1,9)}=8.14, p=0.019\right)$ and no interaction between them $(p>0.6)$.

Face-selective areas. Contrary to the pattern of results found in experiment I using whole-body stimuli, face-selective areas FFA and OFA showed no adaptation effect to headless bodies, and adaptation level did not differ between upright and inverted conditions (Fig. 4B). A repeated-measures ANOVA on the response of the face-selective areas, with area (FFA, OFA), hemisphere, orientation and adaptation as within-subject factors, revealed no main effects of adaptation $(p>0.8)$ or orientation $(p>0.2)$, and no interaction between them $(p>0.8)$. A significant interaction between hemisphere and orientation $\left(F_{(1,7)}=19.53, p=\right.$ 0.003 ) indicates a higher response to inverted than upright bodies in the left-hemisphere compared with no difference between upright and inverted responses in the right-hemisphere. Additionally, we found an interaction between area and hemisphere $\left(F_{(1,7)}=11.72, p=0.011\right)$, with the OFA showing a higher response to bodies in the right than in the left hemisphere compared the opposite pattern (of higher response to bodies in the left than in the right hemisphere) in the FFA.

Object general areas (LOC). As with whole-body stimuli, the LOC exhibited a higher response to inverted relative to upright headless bodies for both same and different pairs, and did not show an adaptation to headless body stimuli (Fig. 4C). A repeated-measures ANOVA of the LOC, with area (LO, pFs), hemisphere, orientation and adaptation as within-subject factors, revealed a main effect of orientation $\left(F_{(1,7)}=13.82\right.$, $p=0.007)$ and no main effect of adaptation $(p>0.4)$. A marginally significant interaction was found between hemisphere and adaptation $\left(F_{(1,7)}=4.38, p=0.075\right)$ with slightly higher adaptation to bodies in the right-LOC. Main effects of area $\left(F_{(1,7)}=9.75, p=0.017\right)$ and hemisphere $\left(F_{(1,7)}=9.53\right.$, $p=0.018)$ revealed higher responses to headless bodies in the LO compared with pFs, and in the right compared with left hemisphere. 
A

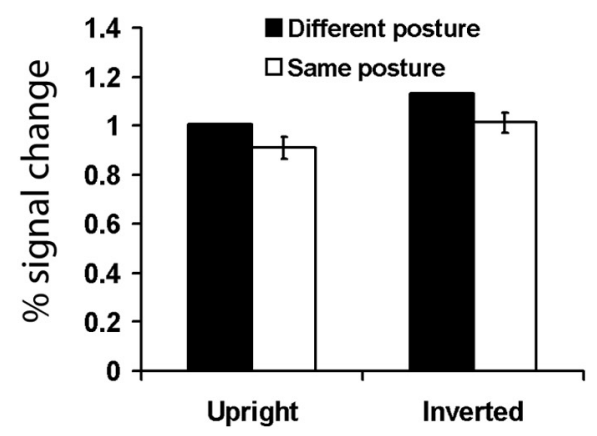

B Face-Selective Areas

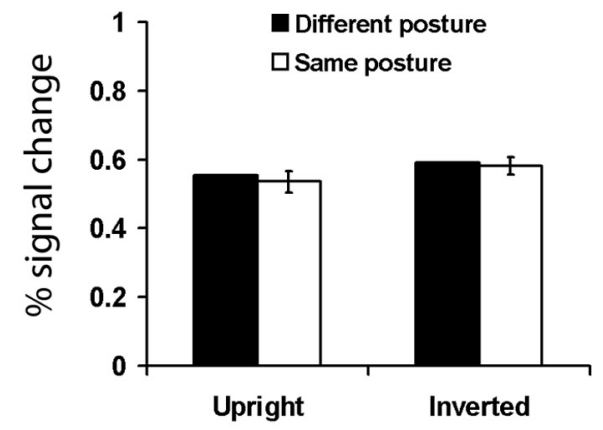

C

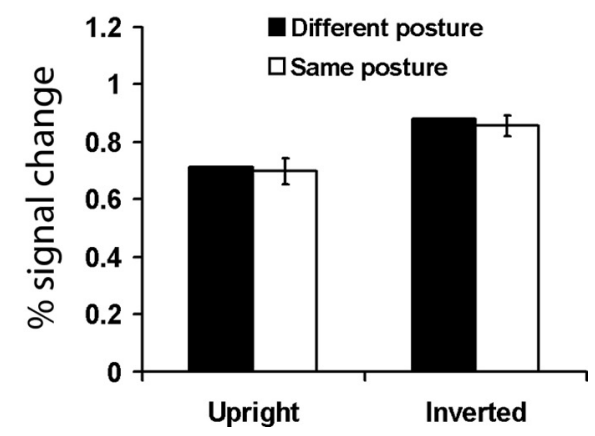

Figure 4. fMR-adaptation to headless bodies. $A$, Body-selective areas showed similar adaptation to upright and inverted bodies and a higher response to inverted than upright stimuli but no interaction between them. This pattern is similar to the response to whole bodies (Fig. $2 A$ ). (see supplemental Fig. 2A, available at www.jneurosci.org as supplemental material for EBA and FBA). $\boldsymbol{B}$, Face-selective areas showed no adaptation to bodies and no difference between the responses to upright and inverted stimuli, in contrast to their response to whole bodies (Fig. 2B) (see supplemental Fig. 2B, available at www.jneurosci.org as supplemental material for OFA and FFA). C, Object general areas showed higher responses to inverted than upright stimuli and no adaptation to bodies. This pattern is similar to the response to whole bodies (Fig. 2C). (see supplemental Fig. 2C, available at www.jneurosci.org as supplemental material for $\mathrm{L} 0$ and pFs). Error bars represent the standard error of the difference between the responses to different and same stimuli (i.e., the standard error of the adaptation effect).

As in experiment I, we examined interactions between category-selective areas after collapsing data across areas and hemispheres. For each category-selective area, ventral and lateral, right and left time courses were averaged based on their relative volumes. We performed a repeated-measures ANOVA with category-selective area, orientation and adaptation as within-subject factors. We separately compared the pattern of response in body areas to that in the face areas and in the object areas.
Comparisons of body versus face-selective areas showed that overall response and adaptation to headless bodies was stronger in the body-selective areas, as reflected in a main effect of category-selective area $\left(F_{(1,9)}=15.90, p=0.003\right)$ and an interaction between category-selective area and adaptation $\left(F_{(1,9)}=12.58\right.$, $p=0.006)$. Whereas body areas showed a higher response to inverted than upright headless bodies, there was no effect of orientation in face-selective areas (a marginally significant interaction between category-selective area and orientation $F_{(1,9)}=4.57, p=$ $0.061)$, despite a main effect of orientation $\left(F_{(1,9)}=5.15, p=0.049\right)$.

Comparisons of body versus object-selective areas uncovered a larger adaptation as well as an overall stronger response to headless bodies in the body-selective areas, seen in an interaction between category-selective area and adaptation $\left(F_{(1,9)}=20.32\right.$, $p=0.001)$ and a main effect of category-selective area $\left(F_{(1,9)}=\right.$ $9.33,0.014)$. In addition, LOC showed a larger orientation effect, with higher response to inverted than upright bodies, compared with the body-selective areas (an interaction between categoryselective area and orientation $\left.F_{(1,9)}=5.95, p=0.037\right)$.

\section{A comparison between whole and headless bodies}

A comparative analysis of the two experiments was performed on the collapsed data across areas and hemispheres. For each category-selective area, ventral and lateral, right and left time courses were averaged based on their relative volumes. We separately compared each area's response patterns in the first experiment to its responses in the second experiment, using a repeated-measures ANOVA with orientation (upright, inverted) and adaptation (same, different) as within-subject factors, and with stimulus (whole-body, headless-body) as a categorical factor.

Body-selective response pattern did not differ between whole and headless bodies (no main effect of stimulus $p>0.075$; no interactions $p>0.5$ ), with an adaptation to both inverted and upright bodies $\left(F_{(1,18)}=21.08, p=0.0002\right)$ and a higher response to inverted than upright body stimuli $\left(F_{(1,18)}=20.06, p=\right.$ 0.0002).

Face-selective response pattern was significantly modified by the type of body stimulus. adaptation was stronger to upright whole bodies in contrast to the lack of such an effect for headless bodies (a marginally significant three-way interaction between adaptation, orientation and stimulus $\left.F_{(1,18)}=3.86, p=0.065\right)$. Additionally, an interaction between orientation and stimulus $\left(F_{(1,18)}=5.86, p=0.026\right)$ shows a higher response to upright than inverted whole bodies but not headless bodies.

Object-selective areas maintained a similar pattern of response across experiments (no effects or interactions of stimulus $p>0.4)$, with no adaptation to bodies $(p>0.1)$ and with a higher response to inverted than upright body stimuli (a main effect of orientation $\left.F_{(1,18)}=38.11, p=0.000008\right)$.

In summary, only the face-selective areas showed higher sensitivity for upright than inverted bodies, which corresponds with the behavioral BIE. Similar to the behavioral BIE, the FFA showed this pattern of response for whole bodies but not for headless bodies. In contrast to the pattern of results found in the faceselective areas, the body-selective areas and object general areas showed comparable activity in response to whole and headless bodies. The body-selective area was sensitive to the posture of both upright and inverted bodies whereas the LOC was not sensitive to either orientation.

\section{Discussion}

In the current study we used fMRI to test whether the BIE is mediated by body-selective brain mechanisms or face-selective 
brain mechanisms. Our findings clearly show that the BIE is mediated by face-selective but not body-selective mechanisms. Only face-selective areas showed higher sensitivity for discrimination of upright than inverted bodies. Similar to the behavioral BIE, this pattern of response of the face-selective areas was found for whole bodies but not headless bodies. In contrast to the faceselective areas, body-selective areas showed similar sensitivity to upright and inverted bodies, regardless of the presence of a head. In contrast to both the face-selective and body-selective areas, object general areas showed no sensitivity to body posture for neither upright nor inverted whole or headless bodies. Our data therefore demonstrate a strong dissociation between the response pattern of the three object-category-selective areas to upright and inverted bodies in which only the response of the face-selective areas corresponds with the behavioral BIE.

The FFA pattern of adaptation to upright but not inverted stimuli, which was found here in response to whole bodies, has also been found by Yovel and Kanwisher (2005) in response to faces, alongside a behavioral face inversion effect (see also Mazard et al., 2006). Importantly, the pattern of response that was found for whole bodies cannot be attributed to the response of these areas to the face itself because faces were fully covered in the whole-body stimuli. The similarity of adaptation pattern between faces and whole faceless bodies in the face areas may therefore indicate that face processing mechanisms are involved in the processing of stimuli from which a face can be inferred even if a face is not physically present in the image. This suggestion is in line with a recent behavioral adaptation study by Ghuman et al. (2010), showing that prolonged viewing of a headless body shifts the perceptual tuning curve for faces, suggesting that an automatic inference about a missing face is sufficient to induce adaptation to faces. These findings may also be related to the fMRI study by Cox et al. (2004), showing higher FFA responses to bodies with degraded faces than to headless bodies presented above degraded faces, indicating FFA selectivity not only to intrinsic facial features, but also to correlated extrinsic features such as contextual body cues. This concept and the response pattern found in faceselective areas suggest that the BIE may result from contextual facial processing in face-selective areas, generated by body cues.

An alternative explanation for the involvement of the face areas in the BIE may be that face-selective brain areas process whole bodies holistically. Because the face inversion effect has been attributed to holistic processing of upright but not inverted faces (Young et al., 1987; Farah et al., 1998; Maurer et al., 2002; Tanaka and Farah, 2003), with the discovery of the BIE, a similar holistic-processing theory was suggested to explain the processing of whole bodies. Supporting findings have demonstrated reduced inversion effect for isolated body-parts and for headless bodies relative to complete bodies (Reed et al., 2006; Minnebusch et al., 2009). The decrease in inversion effect for headless bodies may be consistent with a holistic interpretation if the BIE is found only for complete bodies but not for incomplete bodies. This hypothesis was refuted by Yovel et al. (2010) who showed that in contrast to the absence of inversion effect for headless bodies, inversion effect for incomplete bodies without an arm or without a leg is as large as the inversion effect for whole bodies. Importantly, the inversion effect and discrimination of upright bodies were significantly reduced not only for headless bodies, but also for discrimination of whole bodies that differ in the posture of arm and leg but were identical in head posture. These results suggest that body posture discrimination heavily relies on information from the head and when such information is missing both performance and the inversion effects are markedly reduced. Based on these results, we suggest that the role of the face-selective areas in the
BIE reflects the central role of the head in the processing of whole bodies rather than holistic processing of whole bodies.

The only fMRI study that directly examined whether bodyselective areas generate a holistic representation of bodies was reported by Taylor et al. (2007). In their study, Taylor et al. (2007) found a step-like increase in FBA response to large body parts (e.g., half-body), relative to small body parts (e.g., finger, arm), whereas EBA showed a linear increase in response as a function of body size. The authors concluded that the FBA but not the EBA may generate a holistic representation of human bodies. In our study, we found a similar pattern of activity in response to upright and inverted bodies in the EBA and FBA, which further highlights the suggestion that the BIE may not be associated with holistic processing of human bodies. Having said that, investigation of the BIE and its neural basis provide only indirect evidence with respect to the question of holistic processing of whole bodies. Future studies that directly examine whether body parts are processed interactively or independently and the extent to which this processing is modulated by the presence of the head are needed to determine whether bodies are represented holistically.

The similar adaptation that we found for inverted and upright bodies in the EBA may be inconsistent with Urgesi et al. (2007), who showed that TMS to the EBA disrupts inverted but not upright body posture discrimination. Notably, the EBA in our study did show a higher response to inverted than upright bodies. These results may suggest that effects of TMS may be associated with the selectivity of the response of a given brain area rather than with its adaptation effect. Indeed, in a recent TMS study Pitcher and colleagues reported a triple dissociation for the processing of face identity, body shape and objects in the lateral face, body and object areas, respectively (Pitcher et al., 2009). This hypothesis should be further examined in future TMS studies.

To conclude, the different patterns of response to upright and inverted bodies that we found in the face-, body-, and object-selective brain areas suggest that each of them play a very different role in the processing of body posture. Only the response of the face-selective areas, but not that of the body or object areas, is modulated by the existence of the head and is consistent with the behavioral BIE. We therefore argue that the BIE is not induced by body processing mechanisms but by face or head processing mechanisms. Further research is needed to determine whether the role of face-selective areas in the BIE reflects a head-derived effect independent from body stimuli, or is an effect of contextual face-processing of body information. Furthermore, future studies are needed to determine the extent to which body processing mechanisms represent upright bodies holistically despite the fact that they are not associated with the inversion effect.

\section{References}

Brainard DN (1997) The Psychophysics Toolbox. Spat Vis 10:433-436.

Cox D, Meyers E, Sinha P (2004) Contextually evoked object-specific responses in human visual cortex. Science 304:115-117.

Downing PE, Jiang Y, Shuman M, Kanwisher N (2001) A cortical area selective for visual processing of the human body. Science 293:2470-2473.

Farah MJ, Wilson KD, Drain M, Tanaka JN (1998) What is "special" about face perception? Psychol Rev 105:482-498.

Ghuman AS, McDaniel JR, Martin A (2010) Face adaptation without a face. Curr Biol 20:32-36.

Grill-Spector K, Malach R (2001) fMR-adaptation: a tool for studying the functional properties of human cortical neurons. Acta Psychol (Amst) 107:293-321.

Grill-Spector K, Henson R, Martin A (2006) Repetition and the brain: neural models of stimulus-specific effects. Trends Cogn Sci 10:14-23.

Maurer D, Grand RL, Mondloch CJ (2002) The many faces of configural processing. Trends Cogn Sci 6:255-260. 
Mazard A, Schiltz C, Rossion B (2006) Recovery from adaptation to facial identity is larger for upright than inverted faces in the human occipitotemporal cortex. Neuropsychologia 44:912-922.

Minnebusch DA, Suchan B, Daum I (2009) Losing your head: behavioral and electrophysiological effects of body inversion. J Cogn Neurosci 21:865-874.

Peelen MV, Downing PE (2007) The neural basis of visual body perception. Nat Rev Neurosci 8:636-648.

Pitcher D, Charles L, Devlin JT, Walsh V, Duchaine B (2009) Triple dissociation of faces, bodies, and objects in extrastriate cortex. Curr Biol 19:319-324.

Reed CL, Stone VE, Bozova S, Tanaka J (2003) The body-inversion effect. Psychol Sci 14:302-308.

Reed CL, Stone VE, Grubb JD, McGoldrick JE (2006) Turning configural processing upside down: part and whole body postures. J Exp Psychol Hum Percept Perform 32:73-87.
Tanaka JW, Farah MJ (2003) The holistic representation of faces. In: Perception of faces, objects and scenes: analytic and holistic processes (Peterson MA, Rhodes G, eds). New York: Oxford UP.

Taylor JC, Wiggett AJ, Downing PE (2007) Functional MRI analysis of body and body part representations in the extrastriate and fusiform body areas. J Neurophysiol 98:1626-1633.

Urgesi C, Calvo-Merino B, Haggard P, Aglioti SM (2007) Transcranial magnetic stimulation reveals two cortical pathways for visual body processing. J Neurosci 27:8023-8030.

Young AW, Hellawell D, Hay DC (1987) Configurational information in face perception. Perception 16:747-759.

Yovel G, Kanwisher N (2005) The neural basis of the behavioral faceinversion effect. Curr Biol 15:2256-2262.

Yovel G, Pelc T, Lubetzky I (2010) It's all in your head: why is the body inversion effect abolished for headless bodies? J Exp Psychol Hum Percept Perform 36:759-767. 